\title{
VERS UNE THÉORIE DES PARATEXTES: IMAGES MENTALES ET IMAGES MATÉRIELLES
}

\author{
Daniel Peraya ${ }^{2}$
}

\section{Introduction}

Qu'est-ce qu'un paratexte? Tout texte didactique -livres, articles et manuels d'enseignement ou de vulgarisation- constitue déjà un message scripto-visuel ${ }^{3}$ mêlant au texte photographies, schémas, sociogrammes, sémantogrammes, graphiques, tableaux, langage formulaire et verbal, etc. Cette définition qui englobe ces différentes réalités empiriques sous le nom générique d'“ illustrations " est par ailleurs largement acceptée par les auteurs: "Les mots et les phrases du texte laissent tout autour un espace libre: le cotexte. Dans cet

1 Ce texte constitue une version résumée et remaniée de l'introduction théorique de D. PERAYA et M. C. NYSSEN, Les paratextes dans les manuels scolaires de biologie et d'économie: une étude comparative, Cahiers de la Section des Sciences de l'Education, Université de Genève (sous presse). Le traitement statistique et l'analyse des données ont été réalisés principalement par M. C. Nyssen alors qu'elle était assistante à TECFA. Qu'elle trouve ici l'expression de ma gratitude pour le travail qu'elle a accompli durant cette période, pourtant difficile pour elle.

2 Maître d'Enseignement et de Recherche à l'Université de Genève (TECFA).

3 J. CLOUTIER, La communication audio-scripto-visuelle à l'heure des self-media ou l'heure d'Emerec, Montréal, Presses de l'Université de Montréal, 1973. 
espace disponible seront introduits des titres, des phrases en marge, des informations périphériques (notes, références, etc.) et des illustrations, cet ensemble constituant le paratexte ${ }^{1 "}$. Pourtant elle ne paraît guère satisfaisante. Tout d'abord, de nombreux scientifiques, enseignants et chercheurs, utilisent couramment les représentations figurées parce qu'ils considèrent que, dans leur discipline, les techniques de traitement et de visualisation de l'information sont le seul mode de représentation possible. Ils se refusent donc légitimement à réduire un instrument de conceptualisation à une simple anaphore de l'information verbale. Pour eux le terme même d'illustration devrait être banni. De plus, cette définition ne s'accorde en rien avec les exigences d'une visée théorique. Car, si l'on s'en tient à leur diversité empirique, les paratextes apparaîtront en effet comme un ensemble hétéroclite d'objets concrets parmi lesquels tout classement ou toute analyse systématique sera difficile, voire impossible. Que peuvent en effet avoir en commun, par exemple, une photographie et un schéma, un dessin et une formule mathématique ? De façon générale, il semble toujours délicat de définir des catégories à partir des objets empiriques qui sont susceptibles de les représenter.

Deux caractéristiques communes à tous les paratextes permettent de retrouver une cohérence théorique: d'une part, la fonction sémiotique -symbolique- puisqu'il s'agit toujours de formes de représentation et, d'autre part, le type de discours dans lequel ils s'inscrivent.

Aussi, nous faut-il replacer l'analyse des paratextes dans le cadre d'une théorie générale des représentations et de la signification, donc dans une perspective sémiotique. Heidt ${ }^{2}$, tout en réfutant l'impossibilité de définir une taxonomie des médias unique satisfaisant à la fois les enseignants, les producteurs et les chercheurs, expliquait déjà la faiblesse théorique des différentes classifications existantes par une argumentation semblable. Il suggérait quant à lui de faire porter le principal effort d'élaboration théorique sur la définition des attributs des médias et sur leur interaction avec les variables liées aux tâches et aux apprenants. Cette exigence fondamentale a d'ailleurs été maintes fois formulée par les chercheurs pour qui les limites de l'analyse des

1 D. JACOBI, "Références iconiques et modèles analogiques dans des discours de vulgarisation scientifique", Informations sur les sciences sociales, 4, 24, 1985, p. 848. Jacobi signale dans cet article que le terme a été utilisé pour la première fois par Martins-Balbar dans son article "De l'objet-texte au texte-objet ", Études de linguistique appliquée, 28, 1977, p. 8-23.

2 E.U. HEIDT, "La taxonomie des médias", Communications, 33, 1981, p. 51-75. 
images à vocation éducative proviendraient du manque d'une théorie générale de l'image à laquelle référer son usage pédagogique ${ }^{1}$. On pourrait citer encore Lowe: "Unfortunately, instructional benefits is typically attribued to diagrams in general in a rather indiscrimminate manner without due regard to their individual characteristics $2 "$. Cette ignorance de la spécificité des médias domine encore la recherche psychocognitive comme le montrent, notamment, les derniers travaux de Laurillard. Malgré tout l'intérêt de sa conception conversationnelle du processus d'apprentissage, lorsque l'auteur tente de présenter une taxonomie critériée des médias pédagogiques, il ne va pas au-delà des conceptions classiques et n'échappe donc pas à cette critique ${ }^{3}$.

Il faut ensuite définir les paratextes du point de vue de la pratique communicative et du lieu d'interaction sociale qui sont les leurs ${ }^{4}$. La pragmatique a montré qu'un discours se définit entre autres critères par son intentionnalité. Les paratextes des manuels scolaires s'inscrivent dans une pratique textuelle qui relève d'une réalité institutionnelle et d'un usage social strictement circonscrits: la communication didactique et pédagogique ${ }^{5}$. Dans ce cas, il s'agit d'une intention didactique générale ${ }^{6}$ dont on sait qu'elle contraint les émetteurs à tenir compte principalement du comportement final qu'ils veulent induire. Autrement dit, le paratexte pédagogique correspond strictement aux images de type fonctionnel": celles qui servent à "communiquer ou à connaître le monde", c'est-à-dire des " images de "communication" ou de "compréhension" à l'exclusion des images ou des compositions artistiques qui relèvent d'une volonté de créativité et d'expression individuelles, donc d'une intentionnalité très différente. Point donc de création artistique, de génie ou d'imaginaire: l'institution scolaire n'accepte l'image que policée, à condition que l'imaginaire puisse à son tour faire l'objet d'une didactique.

1 Ibidem; P. Duchastel, M. Fleury \& G. Provost, "Rôles cognitifs de l'image dans l'apprentissage scolaire", Bulletin de Psychologie, XLI, 1988, 386, p. 668-671.

2 R.K. Lowe, "Constructing a mental representation from an abstract technical diagram", Learning and instruction, 20 (3), 1993, p. 157-179.

3 D. LAURILlaRD, Rethinking University Teaching, London/New York, Routledge, 1995.

4 J.-P. Bronckart, Le fonctionnement des discours, Neuchâtel, Delachaux et Niestlé, 1985.

5 D. PERAYA, "Les formes de communication pédagogique médiatisée: le socioéducatif et le didactique", Journal de l'enseignement primaire, 44, mai/juin 1993, p. 24-28.

6 D. PERAYA, La communication scalène, Berne, Peter Lang, 1989.

7 A. MOLES, L'image communication fonctionnelle, Tournai, Casterman, 1981, p. 90. 
Il est vrai que parmi les différents paratextes, l'image réaliste jouit d'un statut particulier, ce qu'atteste son utilisation persuasive et/ou éducative fort ancienne. On connait les ouvrages classiques de Jean Amos Comenius (1592-1671), La grande didactique ou son Orbis Pictus, préfiguration de nos manuels illustrés. Pourtant ce n'est là qu'un des exemples les plus connus. Des ouvrages récents consacrés à l'histoire des formes figuratives et aux modes de représentation tant pré-technologiques que technologiques ${ }^{1}$ soulignent combien, depuis la plus haute antiquité, la catoptrique a suscité de l'intérêt et comment les jeux de miroirs, la magie parastatique et les spectacles d'ombres et de lumières, bien qu'utilisés aux fins d'illusion et de simulation, comportaient une importante dimension éducative ou persuasive. L'intuition de la force et de la prégnance de la vision, et donc de l'image, exprime le sentiment naïf ou naturel selon lequel les spectacles visuels sont, du point de vue de leur perception, bien plus efficaces que le langage verbal. Celui-ci introduirait au sens tandis que les représentations visuelles naturalisantes, les simulacres, donneraient accès au monde, ou en tous cas à certains de ses aspects. Nous pensons qu'il y aurait à cela une première raison: la perception et la décodification de spectacles artificiels (la représentation de personnes, d'objets, de paysages, etc.) recourent à des mécanismes identiques à ceux qui gouvernent la contemplation et la compréhension de spectacles naturels (personnes, objets, paysages, etc.). Nous reviendrons de façon détaillée sur ce point essentiel par la suite.

Traditionnellement, l'image a donc été utilisée, d'une part en raison de son pouvoir de conviction et de désignation et, d'autre part, à cause de sa capacité supposée à faciliter les apprentissages. Mais il est une autre raison de l'intérêt des éducateurs pour l'image: sa fonction vicariale. En effet, l'école souvent organise son enseignement en s'excluant du monde, mais elle permet cependant à celui-ci d'entrer dans ses classes, sous la forme d'images. Tel est aussi le mythe fondateur de la télévision, cette "fenêtre ouverte sur le monde " qui projette chaque foyer, chaque famille, chaque individu au centre des événements et de l'Histoire.

Pourtant l'image a de tous temps donné naissance à un comportement paradoxal: utilisée par les scientifiques et les pédagogues

1 J. PERriault, Mémoire de l'ombre et du son, Paris, Flammarion, 1981; IDEM, La logique de l'usage. Essais sur les machines à communiquer, Paris, Flammarion, 1989; R. ChENAIS, Les racines de l'audio-visuel. Esquisse d'une histoire de la figuration et la représentation en Occident, Paris, Anthropos, 1990. 
-historiquement, le scientisme s'est développé en même temps que le courant illusionniste ${ }^{1}-$, elle fait simultanément l'objet de la méfiance et des uns et des autres. On se souviendra que, à la suite de Bachelard ${ }^{2}$, l'image a longtemps été considérée comme un obstacle à l'accès à une véritable pensée scientifique: la figuration d'une notion ou d'un concept, par essence abstrait, apparaissait comme un substitut dérisoire et une représentation suspecte pour les scientifiques, comme un véritable obstacle épistémologique. A la même époque, Tardy ${ }^{3}$ tentait d'expliquer pourquoi l'image cristallisait l'opposition de la majorité des pédagogues et du corps enseignant: productrice de mirages et d'illusions mais aussi dissipatrice, elle susciterait plaisir et comportements ludiques, incompatibles avec une certaine conception puritaine de la rigueur méthodologique et du travail scolaire. Pour son pouvoir de persuasion et de facilitation, l'image intéresse, mais -on l'a maintes fois écrit- parce qu'elle déborde nécessairement vers l'imaginaire et qu'elle suscite la fascination bien plus que de raison, elle suscite aussi la défiance. Enfin, on ne peut oublier les fortes réticences de Piaget ${ }^{4}$ qui ont vraisemblablement longtemps pesé sur la pédagogie de l'audiovisuel. En 1969, il écrivait: “

Une pédagogie fondée sur l'image, même renforcée par le dynamisme apparent du film, demeure inadéquate à la formation du constructivisme opératoire, car l'intelligence ne se réduit pas aux images d'un film. En bref, l'image, le film, les procédés audiovisuels, dont toute une pédagogie voulant se donner l'illusion d'être moderne nous rebat aujourd'hui les oreilles, sont des auxiliaires précieux à titre d'adjuvants ou de béquilles spirituelles et il est évident qu'ils sont en net progrès par rapport à un enseignement purement verbal. Mais il existe un verbalisme de l'image comme un verbalisme du mot et, confrontés avec les méthodes actives, les méthodes intuitives ne font que substituer, lorsqu'elles oublient le primat irréductible de la recherche personnelle ou autonome du vrai, ce verbalisme plus élégant et plus raffiné au verbalisme traditionnel ${ }^{5}$.

1 J. Perriault, op. cit.

2 G. B ACHELARD, La formation de l'esprit scientifique, Paris, Vrin, 1965.

3 M. TARDY, Le professeur et les images, Paris, PUF, 1966 [2nde éd. revue, 1973].

4 On trouvera une brève analyse des positions respectives de Bachelard et de Piaget dans M. LINARD \& I. PRAX, Images vidéo, images de soi: Narcisse au travail, Paris, Dunod, 1984, p. 199 et ss.

5 J. PIAGET, Psychologie et pédagogie, Paris, Denoël-Gonthier, 1969, p. 110. 
Mais en même temps, cette méfiance n'empêche pas la renaissance, dès les années 60, d'un intérêt théorique pour l'image et son usage pédagogique. Dans le contexte de la linguistique appliquée et des méthodes audiovisuelles ${ }^{1}$ d'apprentissage d'une langue seconde qui sont à l'époque en plein essor, se développe une première réflexion sur les fonctions de l'image et sur son rôle de médiateur intersémiotique $^{2}$. La sémiologie naissante après s'être intéressée aux figures mythologiques ou emblématiques de la société de consommation $^{3}$ prend pour objet les langages non verbaux: le langage de l'image et la publicité ${ }^{4}$, le cinéma ${ }^{5}$, la mode ${ }^{6}$, les objets ${ }^{7}$ ou l'architecture ${ }^{8}$. Le principal débat de l'époque porte sur la nature des différents signes, sur leur typologie et sur la capacité du modèle linguistique à s'appliquer aux autres systèmes de signes ${ }^{9}$. Dans une perspective didactique et à propos des émissions de télévision éducative, on ne peut oublier les analyses de G. Jacquinot ${ }^{10}$. Pour l'image fixe, d'importantes recherches ont été menées dès les années 80, afin de définir le rôle de l'image dans l'apprentissage et dans la littérature de

1 Nous avons opté pour l'orthographe actuelle, audiovisuel, mais nous avons conservé celle des textes originaux dans les citations.

2 A.J. Greimas, "Observations sur la méthode audio-visuelle de l'enseignement des langues vivantes", Études de linguistique appliquée, 1, 1962, p. 137-155; P. RIVENC, "Vers une approche sémiotique du « discours audio-visuel » dans les méthodes d'apprentissage linguistique", Journal de psychologie normale et pathologique, I-2, janvier/juin 1973, p. 189-207; D. PERAYA, "Image et langue. Le langage de l'image", Revue belge de psychologie et de pédagogie, 44, 1982, p. 182; A. LIEURY et al., "Représentations imagées et apprentissage d'un vocabulaire étranger", Bulletin de Psychologie, XLI, 386, 1988, p. 701-709.

3 R. BARTHES, Mythologies, Paris, Ed. du Seuil, 1957.

4 R. BARTHES, "Rhétorique de l'image", Communications, 4, 1964, p. 91-134; G. DURAND, "Rhétorique et image publicitaire", Communications, 15, 1970, p. 7095; G. PENINOU, L'intelligence de la publicité, Paris, Éd. Laffont, 1972.

5 C. METz, "Le cinéma: langue ou langage", Communications, 4, 1964, p. 52-90; IDEM, Essais sur la signification au cinéma, I, Paris, Klincksieck, 1968; IDEM, Essais sur la signification au cinéma, II, Paris, Klincksieck, 1972.

6 R. BARTHES, Le système de la mode, Paris, Éd. du Seuil, 1967.

7 J. BAUDRILlARD, Le système des objets, Paris, Gallimard, 1968; IDEM, La société de consommation, Paris, SGPP, 1970; IDEM, Pour une critique de l'économie politique du signe, Paris, Gallimard, 1972.

8 F. CHOAY, L'urbanisme, utopies et réalités. Une anthologie, Paris, Éd. du Seuil, 1965; U. Eco, La struttura assente, Milan, Bompiani, 1968; D. PERAYA, "En guise d'introduction à une sémiotique architecturale: la problématique de la fonctionsigne", Recherches sociologiques, 2, 1975, p. 149-168.

9 R. BARThES, "Éléments de sémiologie", Communications, 4, 1964, p. 40-51; C. METZ, "Le cinéma...", op. cit.; U. Eco, La struttura assente, op. cit.

10 G. JACQUINOT, Image et pédagogie, Paris, PUF, 1977. 
vulgarisation scientifique ${ }^{1}$. Enfin il y a les travaux psychologiques sur les images mentales, la valeur d'imagerie du lexique, le degré de figurabilité des concepts ou encore les mécanismes psychologiques de compréhension de l'image qui trouvent leurs prolongements aujourd'hui dans les recherches sur les modèles mentaux ${ }^{2}$.

C'est à l'analyse et à l'articulation de certains de ces courants que sera consacrée la suite de ce texte.

1 P. ARNAUd, "Schémas et représentations en chimie dans leurs rapports à la didactique", in GIORDAN, MARTINAND (Ed.), Signes et discours dans l'éducation et la vulgarisation scientifique, Chamonix, Centre Jean Franco, 1984, p. 107-115; J.F. VeziN \& L. VezIN, "Schématisation et exemplification, Signes et discours dans l'éducation et la vulgarisation scientifique", in GroRdan et MARTINAND (Éd.), op. cit., p. 611-618; J.-F. VEZIN, "Mise en relation de schémas et d'énoncés dans l'acquisition de connaissances", Bulletin de Psychologie, XXXVIII, 368, 1985, p. 71-80; J.-F. VEZIN \& L. VEZIN, "Illustration, schématisation et activité interprétative", Bulletin de Psychologie, XLI, n 386, 1988, p. 655-666; D. JACOBI, "Figures et figurabilité de la science dans les revues de vulgarisation scientifique", Langages, 75, 1984, p. 23-41; IDEM, "Références iconiques..., op. cit.; IDEM, Les images de la vulgarisation et la figurabilité de la science. Textes et images de la vulgarisation scientifique, Peter Lang, 1987, p. 109-166; IDEM, "Les images et la vulgarisation scientifique", Bulletin de Psychologie, XLI, 386, 1988, p. 560-570; D. REID, "The picture superiority effect and biological education", Journal of Biological Education, 18, 1984, p. 26-29; IDEM, "Quelques investigations sur le rôle des images dans l'apprentissage à partir de textes de biologie", Les formes du savoir dans les manuels scientifiques, Les cahiers du CRELEF, 28, 1-2, 1989, p. 73-100; J.-P. Astolfi, Y. Ginsburger-Vogel et B. PETERFAlvi, "Aspects de la schématisation en didactique des sciences", Bulletin de Psychologie, XLI, 386, 1988, p. 696-700; P. Duchastel, op. cit.; J. Balllé et S. MAurY, (Ed.), "Les représentations graphiques dans l'enseignement et la formation", Les sciences de l'éducation pour l'ère nouvelle, 1-3, 1993; E. DE CORTE, (Ed.), "Comprehension of graphics in Texts", Learning and Instruction, vol. 3, n³, 1993.

2 A. PAIVIO, Mental representation: A dual coding approach, New York, Oxford University Press, 1986; M. DENIS, Les images mentales, Paris, P.U.F., 1979; IDEM, Image et cognition, Paris, P.U.F., 1989; IDEM \& M. DE VEGA, "Modèles mentaux et imagerie mentale", in M. F. EHRLICH (Ed.), Les modèles mentaux. Approche cognitive des représentations, Paris, Masson, 1993; H. BaRlow, Images and Understandings, Cambridge, Cambridge University Press, 1990; J.-M. GRAILET, "L'amorçage visuel d'objets et les théories de la reconnaissance", Perceptual Priming, XXXII-1, 1992, p. 107-140; P. N. JoHNSON-LAIRD, "Mental models in cognitive science", Cognitive Science, 4, 1980, p. 71-115; IDEM, Mental models: Towards a cognitive science to language, inference, and consciousness, Cambridge, Cambridge University Press, 1983. 


\section{L'analyse sémio-pragmatique}

Nous ne présenterons pas ici l'inventaire détaillé des recherches sémiotiques et pragmatiques qui ont contribué à l'analyse de l'image pédagogique pas plus que leur évolution. Si dans le contexte des sciences de l'éducation, ce rappel s'avère un préalable indispensable, pour un public familier des sciences de la communication, il ne se justifie guère. Le lecteur intéressé pourra cependant se reporter au numéro des Cahiers de la Section des Sciences de l'Éducation ${ }^{1}$. Nous soulignerons cependant quelques perspectives intéressantes qui renouvellent la problématique en contribuant à l'articulation de la sémiotique visuelle avec une théorie cognitive des représentations.

La sémiotique, parce qu'elle étudie les processus de sémiotisation, a pour objet fondamental la diversité des différents systèmes de signes, leur classement ainsi que l'analyse du modus significandi de chacun d'entre eux. Parmi ceux-ci, l'image et les codes iconiques ont occupé une place importante: parce qu'ils présentent un mode de fonctionnement irréductible à celui des signes linguistiques, ils ont fait surgir un grand nombre de problèmes théoriques et méthodologiques. Parmi ceux-ci, la définition du signe visuel et la nature de l'analogie.

Un signe iconique, on l'a dit, possède certaines propriétés de l'objet représenté. Certes, cette définition satisfait notre bon sens, mais n'est-elle pas trompeuse? Que peut bien signifier "avoir les mêmes propriétés"? Dans la perspective strictement structurale de l'époque, Eco a défendu la thèse alors passée quasiment inaperçue, selon laquelle la communication prendrait naissance non pas dans le rapport entre le code et le message mais dans les mécanismes mêmes de la perception qui fondent les modalités de production des signes: "les signes iconiques ne possèdent pas les propriétés de l'objet représenté, mais ils reproduisent certaines conditions de la perception commune sur la base des codes perceptifs normaux et par la sélection des stimuli qui -ayant éliminé d'autres stimuli- peuvent me permettre la construction d'une structure perceptive. Cette structure perceptive possède -sur la base des codes de l'expérience acquise- la même signification que l'expérience réelle dénotée par le signe iconique "2.

1 D. PERAYA \& M.-C. NYSSEN, Les paratextes dans les manuels scolaires de biologie et d'économie Une étude comparative, Genève, Cahiers de la Section des Sciences de l'Education, nº 78, FPSE, décembre 1995.

2 U. Eco, "Sémiologie des messages visuels", Communications, 15, 1970, p. 14. 
Autrement dit encore, "si le signe [iconique] a des propriétés communes avec quelque chose, il les a non avec l'objet mais avec le modèle perceptif de l'objet; il est constructible et reconnaissable d'après les mêmes opérations mentales que nous accomplissons pour construire le perçu, indépendamment de la matière dans laquelle ces relations se réalisent". Autrement dit, Eco situe la relation analogique non pas entre la représentation -le signe iconique- et l'objet représenté -l'objet réel- mais bien entre l'icone et "un modèle perceptif de l'objet" 2 .

C'est sur la base de telles hypothèses que le Groupe $\mu$ fonde son Traité du signe visuel. Pour les auteurs, l'une des premières tâches consiste à établir les fondements perceptifs d'une sémiotique visuelle, autrement dit à analyser le processus sensation vs perception vs cognition. Le système visuel produirait dans les trois modalités que retiennent les auteurs -spatialité, texture et chromatisme- des structures de percepts élémentaires, intégrant et organisant les stimuli à partir de structures spécialisées: extracteurs de motifs, de directions, de contrastes, etc. On obtiendrait ainsi la production de figures puis de formes et enfin d'objets. Les figures naissent d'un processus "d'équilibration des zones d'égalité de stimulation"-d'où les notions de champ, de limite, de ligne, de contour, etc.- tandis que les formes font " intervenir la comparaison entre diverses occurrences successives d'une figure et mobilise[ent] donc la mémoire"3. Le passage à l'objet interviendrait ensuite par l'adjonction de propriétés non visuelles provenant des autres modalités sensorielles au moment où la forme se doterait de caractéristiques permanentes. L'objet qui s'apparente à la notion de type est alors considéré comme fort proche de celle du signe: "De ce que les objets sont une somme de propriétés, douées de permanence et guidant l'action, on peut avancer que cette notion rejoint celle de signe. Le signe est en effet, par définition, une configuration stable dont le rôle pragmatique est de permettre des anticipations, des rappels ou des substitutions à partir de situations. Par ailleurs le signe a, comme on l'a rappelé, une fonction de renvoi qui n'est possible que moyennant l'élaboration d'un système."4. Le modèle du décodage visuel peut alors prendre la forme suivante :

1 Ibidem, p. 21.

2 Ibidem.

${ }^{3}$ Groupe $\mu$, Traité du signe visuel. Pour une rhétorique de l'image, Paris, Éd. du Seuil, 1992, p. 68.

4 Ibidem, p. 81. 


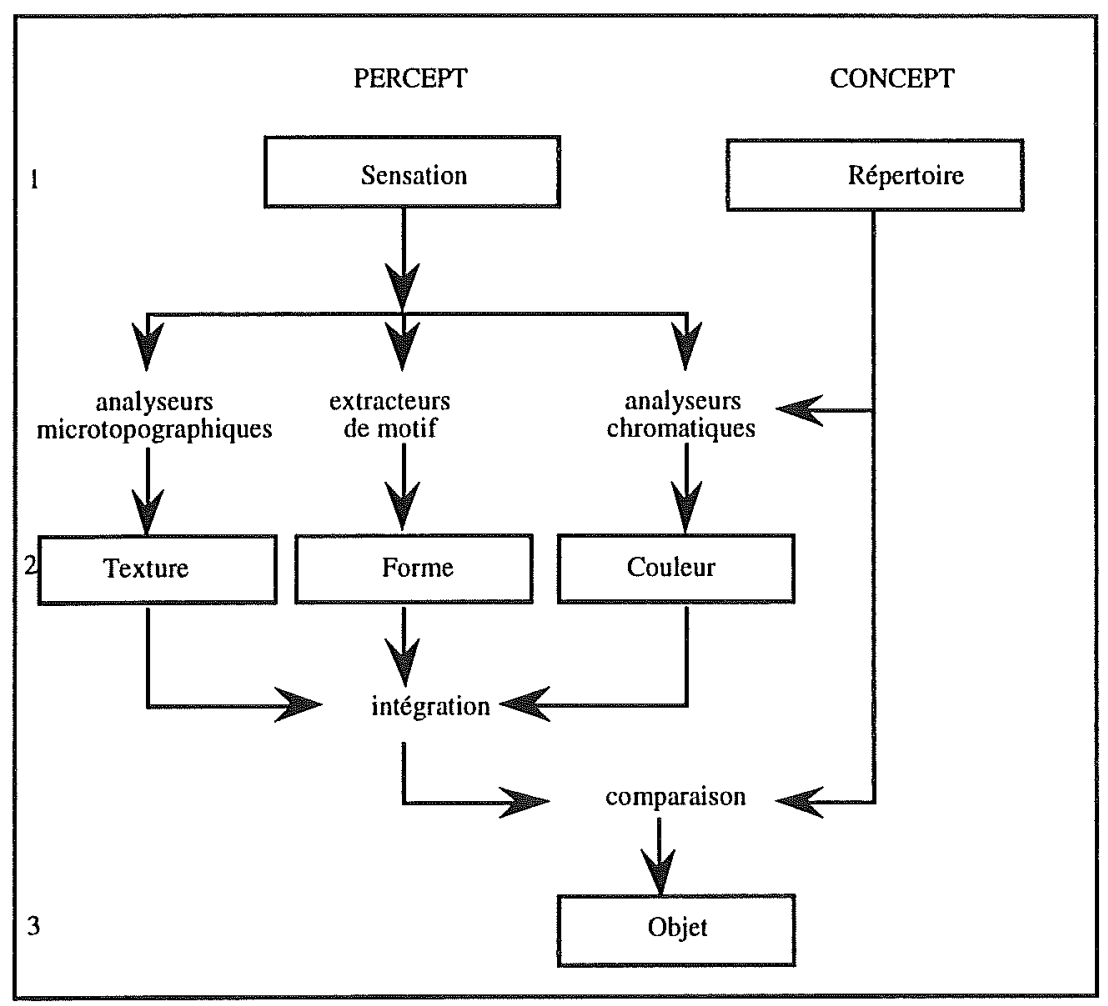

Schéma 1: Un modèle du décodage visuel ${ }^{1}$.

Mais il est évident que la relation analogique de même que la conception du signe doivent alors être profondément modifiées. D'une unité bipartite, les auteurs font une unité tripartite composée du signifiant, du référent et du type. Les définitions de chacun de ces trois pôles et de leurs rapports respectifs propose une nouvelle approche de l'analogie et de la relation iconique qui, poussée à l'extrême, débouche sur deux impasses théoriques bien connues. La première consiste à dire que tout objet est l'icône de lui-même, ce qu'Eco formulait déjà sous la forme d'une boutade lapidaire: "L'icone la plus parfaite de mon nez, c'est mon propre nez...". L'autre permet d'affirmer que tout objet peut être considéré comme l'icone de tout autre objet, car il est toujours possible de trouver un certain aspect qui les rassemble....

1 Ibidem, p. 91. 
Reprenons brièvement ces définitions. Le référent est "l'objet entendu non comme somme inorganisée de stimuli, mais comme membre d'une classe (ce qui ne veut pas dire que ce référent soit nécessairement réel). L'existence de cette classe d'objets est validée par celle du type.". Le type par contre est une classe conceptuelle, abstraite: "Par exemple, le référent du signe iconique chat est un objet particulier, dont je puis avoir l'expérience, visuelle ou autre, mais il n'est référent qu'en tant que cet objet peut être associé à une catégorie permanente: l'être-chat." Le signifiant, enfin, "est un ensemble modélisé de stimuli visuels correspondant à un type stable, identifié grâce à des traits de ce signifiant, et qui peut être associé à un référent reconnu, lui aussi, comme hypostase du type; il entretient avec ce référent des relations de transformations"!

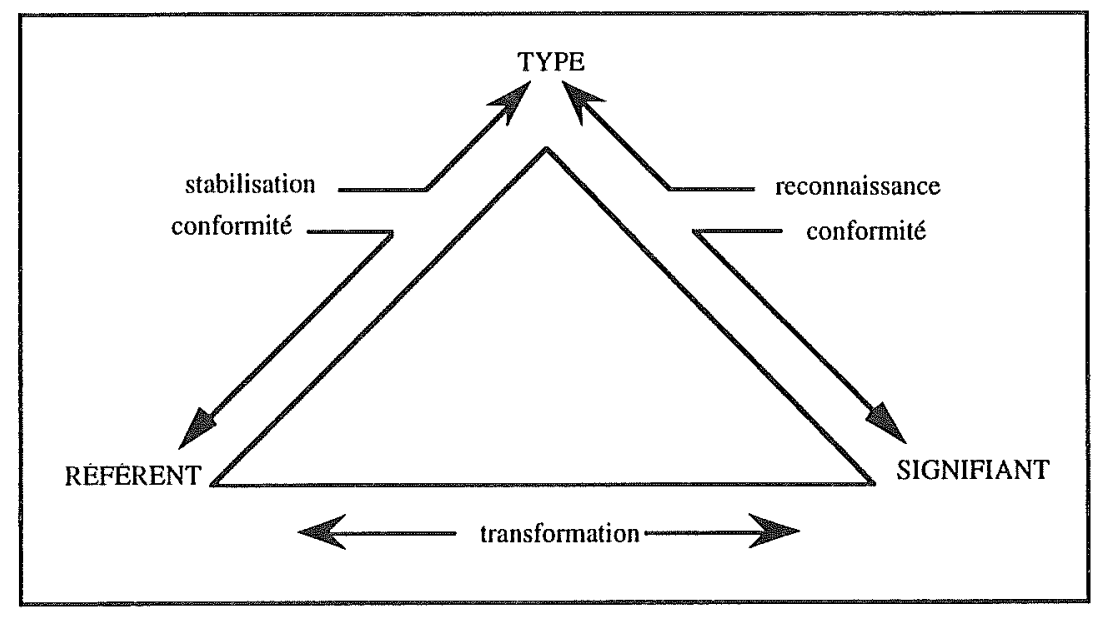

Schéma 2: Le signe iconique ${ }^{2}$

Deux aspects nous intéressent directement dans ce modèle: tout d'abord, la notion de transformation qui permet de fonder, par exemple, les échelles d'iconicité sur une base théorique et formelle; ensuite, la notion de type. Il sera en effet intéressant de replacer ce modèle dans le cadre des études sur l'imagerie mentale et sur les modèles mentaux que nous évoquerons par la suite, puisque le type ainsi défini s'apparente à une représentation mentale de type analogique. A ce propos, notons déjà que la notion de monosémie ne peut

1 Ibidem, p. 136-137.

2 Ibidem, p. 136. 
se construire que sur la base d'hypothèses de cette nature: une image en effet sera interprétée de façon univoque par plusieurs sujets à la condition que ceux-ci partagent une représentation commune et plus l'image sera conforme à ce modèle, plus elle apparaîtra monosémiquel. En conséquence, la notion de conformité au type implique que l'on reconnaisse l'importance de la familiarisation du sujet avec les stimuli et donc aussi les déterminants culturels du processus "perception vs cognition".

C'est dans cette perspective que l'on pourrait rattacher les travaux de certains auteurs comme Leclercq et Rombaux: ceux-ci étudient expérimentalement les conditions de monosémie de l'image -dessin au trait, photographie détourée ou non, etc.- et proposent différents indices permettant de quantifier, par exemple, son pouvoir évocateur ou son taux d'efficacité monosémique. Ces différents indices n'ont bien sûr d'autre pertinence que relative; ils permettent de comparer entre elles plusieurs représentations du même référent afin de déterminer celle qui est la plus fréquemment interprétée correctement avec un degré de certitude maximal. On ne s'étonnera pas que les représentations les plus efficaces ne soient pas nécessairement les plus réalistes: une surcharge d'information peut en effet nuire à l'identification du type. Rappelons à ce propos les résultats d'une expérience réalisée au Népal par Richaudeau ${ }^{2}$ qui met en évidence le taux de reconnaissance de représentations selon leurs différentes modalités :

- dessin ombré: $72 \%$;

- photographie détourée: $67 \%$;

- dessin (ligne): 62\%;

- dessin à-plat noir: $61 \%$;

- photographie: $59 \%$;

- dessin stylisé: $49 \%$.

Ces chiffres n'ont cependant de valeur qu'indicative car ils représentent la moyenne des taux de reconnaissance pour chaque modalité de trois représentations différentes: un canari (une sorte de cruche), une fillette portant un canari et un mouton. Or, la nature de l'objet représenté semble avoir, elle aussi, une grande importance sur le score

1 D. LeClerCQ \& RombauX, cité in D. LeCLeRCQ, La monosémie de l'image, Audiovisuel et apprentissage, Liège, Université de Liège, Service de Technologie de l'Education, 1989.

2 F. RICHAUDEAU, Conception et production des manuels scolaires, Paris, Retz/Unesco, 1979, [2nde édition, 1981]. 
de reconnaissance, parfois plus grande que la modalité de représentation elle-même. Par exemple, la photographie de la fillette portant une cruche obtient un score de reconnaissance de $82 \%$ alors que celles du mouton et du canari obtiennent respectivement, $29 \%$ et $28 \%$. Nous rapprocherons cette observation de l'effet de l'objet connu en psychologie expérimentale ou encore de certains résultats expérimentaux qui font état, au niveau conceptuel, d'un effet de focalisation d'attention et de mise au premier plan:

Étant donné deux entités conceptuelles, toutes choses égales par ailleurs, il existe des facteurs qui tendent à focaliser l'attention sur certaines entités plutôt que sur d'autres. Par exemple, la focalisation est généralement privilégiée sur les objets animés plutôt que sur les objets non animés, sur les humains plutôt que sur les non humains, sur les personnages principaux plutôt que sur les personnages secondaires ${ }^{1}$.

Les modalités de représentation ont leur importance et favorisent l'identification et la reconnaissance de l'objet représenté: la conformité de la représentation au type, tel que le définit le Groupe $\mu$, constitue l'une des conditions premières de sa reconnaissance, et donc de son acceptabilité. Toutes les recherches convergent aujourd'hui pour souligner le rapport entre représentations matérielles et représentations mentales, entre images et modèles mentaux. C'est pourquoi il nous semble important de reconnaître la nécessité théorique d'une articulation entre une sémiotique des représentations et une théorie psychologique de ces dernières.

\section{Les images et les modèles mentaux}

L'activité de représentation est liée à la fonction symbolique, c'est-à-dire à cette forme de l'activité humaine qui consiste à produire des symboles dont la caractéristique est de tenir lieu d'autres entités. Tout symbole, rappelons-le, est en effet un "valant pour ". Autrement dit,

Il y a activité de représentation lorsqu'un objet ou lorsque les éléments d'un ensemble d'objets se trouvent exprimés, traduits, figurés, sous la forme d'un nouvel ensemble d'éléments, et

1 M. DENIS \& M. DE VEGA, op. cit., p. 97. 
qu'une correspondance systématique se trouve réalisée entre l'ensemble de départ et l'ensemble d'arrivée!.

Cette mise en correspondance fait intervenir deux aspects: la conservation des relations entre les éléments faisant l'objet de la représentation, d'une part, et la transformation de l'information de départ par un processus de codage qui donne lieu à un changement de nature, d'autre part.

Producteur de symboles, l'être humain crée des représentations qui laissent des traces matérielles de type iconographique ou picturale, extérieures à l'individu qui les a produites. Mais il existe également des représentations cognitives de nature psychologique qui, en plus des caractéristiques générales de toutes représentations, posséderaient des propriétés spécifiques. En réalité, ces représentations cognitives reflètent ce que l'individu retient de ses interactions avec le monde et constituent " une fonction générale d'intégration mentale active de nos relations externes et internes au réel "2. Enfin, tentant de clarifier les différentes acceptions du terme "représentation", Denis distingue encore l'activité de représentation et les entités cognitives produites par cette activité, autrement dit la représentation proprement dite et le processus qui l'engendre. Cette distinction fondamentale entre produit et processus, nous le verrons, n'est pas toujours respectée par les pédagogues qui souvent assimilent l'un à l'autre dans une même analyse ${ }^{3}$.

Quant aux fonctions générales des représentations -objets matériels ou produits cognitifs--, rappelons-les brièvement. Elles permettent, selon Denis ${ }^{4}$, de conserver l'information amenée à se détériorer et dont l'accès nous sera progressivement rendu difficile par la suite: la photographie familiale ou touristique, par exemple, assume fort bien ce rôle. Mais les représentations rendent aussi accessibles des informations qui ne le sont pas dans les conditions normales, "naturelles ", de perception: c'est le cas, par exemple, des schémas de câblage d'un appareil électronique, des photos de la face cachée de la lune ou encore de l'imagerie médicale.

1 M. DenIS, Image et cognition, Paris, P.U.F., 1989, p. 21.

2 M. LINARD et I. PraX, op. cit., p. 204.

3 Denis distingue encore les représentations disponibles et les représentations actualisées et, parmi ces dernières, celles qui le sont sans une réelle expérience cognitive et celles qui sont attestées par l'individu (M. DENIS, Image et cognition, op. cit.).

4 Ibidem, p. 25-27. 
A cette fonction vicariale s'ajoute une fonction d'explicitation de l'information. A ce titre les représentations constituent des substituts partiels dans la mesure où " ils ne sont susceptibles de remplacer les objets originaux que pour certaines fonctions, principalement de nature cognitive". Ensuite, puisque l'information contenue dans les représentations est utilisée lors de l'exécution de nombreuses tâches, elles sont considérées " comme des instruments de guidage, d'orientation, de régulation des actions d'un individu ": un plan urbain, un plan d'architecte, le schéma de montage d'une maquette, etc. sont autant d'illustrations aujourd'hui banalisées de cette utilisation fonctionnelle des représentations, principalement des schémas. On sait, de plus, que les représentations peuvent assurer une fonction de systématisation d'un corpus; à ce titre, elles constituent un instrument de description et de connaissance du réel: l'auteur mentionne, par exemple, un arbre généalogique, les relations d'appartenance à des classes dans une taxonomie, les organigrammes, les " fonctionnigrammes ", etc. Quant aux symboles du code de la route, aux pictogrammes internationaux, ils ont quant à eux une fonction de signalisation que fonde une intention de communication. Enfin, d'autres représentations assument diverses fonctions, par exemple métaphorique ou emblématique: ces représentations font alors partie de systèmes plus complexes d'échanges d'informations, interindividuels ou sociaux.

La psychologie qui s'intéresse à la notion de représentation cherche donc à rendre compte du fonctionnement des systèmes qui peuvent recueillir, analyser et garder l'information sous une forme symbolique pour ensuite en tirer avantage lors de conduites ou de l'exécution de tâches. Mais parler des représentations et de leur contenu ne suffit pas, il faut également envisager la forme ou les formes sous laquelle (ou lesquelles) ces représentations sont inscrites dans le système cognitif de l'individu. De nombreux arguments ont été avancés en faveur d'un codage strictement linguistique des représentations: nous possédons des représentations linguistiques et le langage naturel semble en effet bien adapté à représenter une grande diversité de faits de nature différente. De plus, l'introspection semble nous conforter dans cette hypothèse puisque nous avons l'impression de penser dans notre langue. L'hypothèse structurale de Saussure, selon laquelle la pensée serait un tout amorphe mis en forme par le double découpage qu'impose l'unité linguistique, s'inspire de cette même conception, mais en même temps la renforce. Il est vrai que le modèle prédicatif possède une validité cognitive puisque toutes les langues 
connues possèdent une structure prédicative: c'est donc un argument important en faveur de ce modèle. Aussi s'accorde-t-on aujourd'hui sur l'existence d'un modèle de codage propositionnel indépendant du langage naturel mais dont " l'unité cognitive de base, qui intervient dans les traitements sémantiques, (...) est la proposition constituée par un prédicat et ses arguments"1. Si certains auteurs comme Pylysphyn² considèrent que c'est là le seul modèle, de nombreux travaux expérimentaux ont mis en évidence l'existence de représentations mentales analogiques.

Nous rappellerons tout d'abord l'ouvrage de Arnheim, La pensée

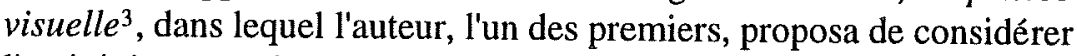
l'activité perceptive comme une activité cognitive à part entière. Actuellement, les chercheurs s'accordent sur l'idée qu'il existe des formes distinctes de représentations cognitives, de connaissances déclaratives: " il existerait donc des systèmes distincts, possédant des règles de fonctionnement différenciées, résultant de genèses différentes assurant des formes de traitement de l'information à travers des codes symboliques distincts"4. Tel est le modèle du double codage ${ }^{5}$. Les activités cognitives seraient en effet régies par deux systèmes de codage différenciés, par deux modes de représentation symbolique: 1) un système de représentations arbitraires, verbales ou propositionnelles, lié à l'expérience du langage qu'a l'individu et 2) un système de représentations figuratives, basé sur une " sémantique de la ressemblance "6 et lié à l'expérience perceptive de notre environnement. Le premier de ces deux systèmes, peu dépendant du caractère concret des situations, conviendrait mieux au traitement de l'abstraction que le

1 J.-F. RICHARD, Les activités mentales. Comprendre, raisonner, trouver des solutions, Paris, Dunod, 1990, p. 185.

2 Z. W. PYLYSHYN, "What's the mind's eye tells the mind's brain: A critique of mental imagery", Psychological Bulletin, 80, 1973, p. 1-24; IDEM, "The imagery debate : Analogue media versus tacit knolewdge", Psychological Review, 88, 1981, p. 16-45.

3 R. ARNHEIM, La pensée visuelle, Paris, Flammarion, 1976, [Édition originale en langue anglaise, 1969].

4 M. DENIS, "Forme imagée de la représentation cognitive", Bulletin de Psychologie, 386, 1988, p. 710-715.

5 A. PaIvio, Imagery and verbal process, New York, Holt, Rinehart \& Winston, 1971; IDEM, Mental representation..., op. cit.; S. M. KosSLYN, "Can imagery be distinguished from other forms of internal representation? Evidence from studies of information retrieval times", Memory and cognition, 4, 1976, p. 291-297; IDEM, Image and mind, Cambridge, MA, Harvard University Press, 1980.

6 M. DENIS et M. DE VEGA, op. cit., p. 89. 
second qui serait mis en oeuvre lorsque les situations à traiter par l'individu se référeraient justement à des événements concrets. Il faut cependant nuancer ce principe. En effet, il n'y a pas de relation nécessaire entre la modalité perceptive, le type de représentation et la forme du codage en mémoire: le langage bien souvent peut être le déclencheur d'une activité d'imagerie, comme c'est le cas pour les mots concrets. En plus de cette spécialisation fonctionnelle qui constituerait indéniablement un des avantages de cette théorie, il en est un autre. L'information étant stockée séparément, la capacité de stockage serait d'autant plus importante. Pourtant, pour tirer avantage du double codage, il faudrait aussi que les deux types d'information produisent des effets de convergence (collaborative effects) et n'entrent donc pas en compétition ${ }^{1}$; enfin, il leur faudrait être en interrelation (richly cross-referenced $)^{2}$.



Schéma 3: La théorie du double codage ${ }^{3}$

1 On rapprochera cette analyse des recherches concernant la charge mentale de travail et la surcharge cognitive fréquente lorsqu'un sujet est amené à réaliser simultanément deux tâches différentes. A titre d'exemple citons les travaux de Sweller et Chandler qui montrent que certains formats de présentation combinant à la fois des schémas et des textes écrits sont traités de manière différente. Dans le cas où les informations (schémas et textes) sont associées en complémentarité, elles vont être traitées comme provenant d'une source unique. Dans le cas inverse, lorsque l'articulation entre les schémas et les textes n'est pas directement compréhensible, les informations vont être traitées comme provenant de deux sources différentes augmentant la charge mentale des sujets. On consultera une brève synthèse de cette problématique chez P. Dillenbourg, Réalisation de logiciels interactifs. Notes provisoires, Genève, TECFA, FPSE, 1992.

2 J. R. KIRBY, "Collaborative and cognitive effect of verbal and spatial processes", in E. DE CORTE, Comprehension of graphics in Texts, Learning and Instruction, Vol. 3, n³, 1993, p. 202.

3 Ibidem, p. 203. 
Cette distinction entre deux types de représentation est importante dans la mesure où elle met en évidence l'existence de systèmes de représentations mentales " analogiques", qui conservent donc les propriétés structurales des objets représentés -certes, avec des variations possibles dans le degré d'analogie-, et d'autres utilisant, quant à eux, des signes arbitraires, chacun de ces systèmes correspondant de ce fait à deux des pôles de la classification classique des signes et des symboles matériels. Notons cependant que la dimension de contiguité vécue propre aux indices n'est pas absente de certaines formes de représentation mentale comme l'avaient déjà noté, par exemple, Wallon et Piaget. Pour ce dernier -on le sait- les images figuratives naissent de l'imitation sensori-motrice active intériorisée sous la forme de schèmes. Plus récemment, Denis et de Vega ${ }^{1}$ mentionnent les résultats d'expériences qui montrent que toutes les dimensions spatiales (haut/bas, gauche/droite, devant/derrière) ne sont pas également accessibles lorsqu'il s'agit pour des sujets de restituer, à partir d'un modèle mental, les relations spatiales entre différents objets. La dimension verticale apparaît prédominante et la plus facile à discriminer dans la mesure où elle implique " deux fortes sources de dissymétrie: la gravité d'une part et la position canonique du corps humain d'autre part "'2. Viennent ensuite les dimensions devant-derrière alors que les dimensions gauche-droite, par manque de traits de différenciation saillants, paraissent difficilement discernables. Et l'auteur de conclure: "Les modèles mentaux ${ }^{3}$ spatiaux sont «biaisés» par les connaissances et par l'ensemble de l'expérience perceptivomotrice qui rendent certaines dimensions plus accessibles que d'autres"4.

Comme d'autres auteurs, Denis auquel nous empruntons cette brève synthèse fonde sa démarche sur l'acceptation d'une filiation entre les activités d'imagerie ${ }^{5}$ et les activités perceptives dont dérivent les premières. Le propos consiste alors à faire apparaître jusqu'à quel

1 M. DENIS et M. DE VEGA, op. cit., p. 88-89.

2 lbidem, p. 87.

3 Sur l'articulation des concepts d'images et de modèles mentaux, on se référera à la contribution de ces deux auteurs, chapitre IIII: "Modèles mentaux et imagerie mentale".

4 Ibidem.

5 Selon Kosslyn, les représentations imagées seraient générées à partir de deux types de représentation de la mémoire à long terme: " des représentations littérales, qui codent l'apparence structurale schématique de l'objet (skeletal incoding), et de représentations propositionnelles, qui codent sous forme abstraite la liste des parties composant l'objet, leurs localisations, les relations de l'objet à l'égard de catégories subordonnées, etc." (M. DENIS, Image et cognition, op. cit., p. 54). 
point existe une similitude entre ces deux types d'activités. Sur la base de nombreux travaux expérimentaux, on s'accorde aujourd'hui à reconnaître l'existence d'une double similitude entre ces deux types d'activités: fonctionnelle et structurale. Fonctionnelle tout d'abord: dans de nombreuses situations expérimentales portant sur des activités de mémorisation, les résultats montrent que les effets d'une présentation perceptive sont comparables à ceux d'une élaboration d'images mentales visuelles: par exemple, lors "d'activités d'apprentissage avec rappel libre, la perception de dessins semble donc avoir, au plan du codage mnémonique, un effet similaire à l'évocation purement mentale des objets correspondants"'. Pourtant, cette similitude fonctionnelle ne s'observe pas uniquement lors de situations expérimentales visant à mesurer la mémorisation: elle s'observe aussi, par exemple, dans le cas de la restitution de propriétés figuratives au cours de jugement de similarité2.

Ainsi, activité perceptive et imagerie produisent les mêmes effets. Pour Denis, l'étape suivante consistera à identifier les caractéristiques qui, partagées par ces différents systèmes de représentation, permettent d'expliquer la similarité des mécanismes fonctionnels: les similitudes structurales entre les images et les percepts jouent ici un rôle essentiel. Il existe aujourd'hui suffisamment de données expérimentales qui attestent de "l'isomorphisme structural des représentations imagées à l'égard des événements perceptifs à partir desquels elles se sont constituées "3: de nombreuses recherches, notamment sur l'exploration mentale, mettent en évidence, d'une part, l'existence d'une structure interne propre aux représentations du type analogique et, d'autre part, un isomorphisme structural entre ces images et les représentations d'origines perceptives, les percepts. Un auteur comme Vergnaud ${ }^{4}$ préfère quant à lui parler d'homomorphisme insistant sur le fait que la relation ne doit pas être bi-univoque; il suffit que "les éléments de l'ensemble d'arrivée correspondent à des classements de l'ensemble de départ ". Quoi qu'il en soit, on peut représenter les rela-

1 Ibidem, p. 67.

2 R.M. SHEPARD et S. CHIPMAN, "Second order-isomorphism of internal representations: Shapes of stoles", Cognitive Psychology, 1, 1970, p. 1-17, cité par M. DENIS (ibidem, p. 68).

3 Ibidem, p. 22.

4 G. VERgnAud, "Signifiants et signifiés dans une approche psychologique de la représentation", in J. BAILLE et S. MAURY (éd.), Les représentations graphiques dans l'enseignement et la formation, Caen, CERSE, Université de Caen, 1-3, 1993, p. 9. 
tions d'analogie entre ces trois objets et les activités correspondantes, de la façon suivante :



Schéma 4: Relations d'analogie (I)

Enfin, cette forme d'analogie structurale se voit généralement complétée par une seconde hypothèse: l'image pourrait en effet faire l'objet de traitements impliquant des processus semblables à ceux qui sont mis en oeuvre pendant l'activité perceptive. La similitude des mécanismes des activités perceptive et imageante semble s'expliquer par plusieurs hypothèses neurophysiologiques et neuropsychologiques: les deux types d'activité posséderaient un substrat neuronal commun et l'imagerie partagerait avec le système visuel certaines structures neuronales ${ }^{1}$; de plus, les représentations activées sur ce substrat par la perception et l'imagerie présenterait de fortes analogies ${ }^{2}$ tandis que les modules de traitement seraient identiques ${ }^{3}$.

Aussi “ l'image par les propriétés structurales qu'elle hérite de la perception est un instrument cognitif permettant à l'individu d'effectuer des calculs, des simulations, des inférences, des comparaisons sans devoir recourir à des systèmes calculatoires formels "4. L'image mentale est en effet le produit d'une activité mais aussi le lieu, un espace d'application de divers processus mentaux. Kosslyn déjà postulait quatre types d'activité applicables aux images: 1) le rafraîchissement du support mental, sorte de buffer visuel, aux

1 H. Barlow, op. cit; J.-P. Changeux, L'homme neuronal, Paris, Fayard, 1993 cité par M. DENIS, Image et cognition, op. cit., p. 96.

2 M. FARAH, "Is visual imagery really visual ? Overlooked evidence from neuropsychology", Psychological Review, 95, 1988, p. 307-317.

3 S. M. KoSSLYN, "Seing and imagining in the cerebral hemispheres: A computational approach", Psychological Review, 94, 1987, p. 148-175.

4 M. DENIs, Forme imagée..., op. cit., p. 714. 
propriétés d'un espace coordonné, sur lequel elle s'inscrivent; 2) les processus d'exploration; 3 ) les processus de transformation tels que la rotation mentale; 4) l'utilisation des images pour répondre à certaines questions portant sur des objets.

Enfin, certains chercheurs ont montré l'existence d'une similarité entre la structure de l'objet et celle de l'image mentale stockée en mémoire permanente ${ }^{1}$. L'image est donc une forme de représentation dont le degré d'abstraction ne fait cependant pas perdre à la représentation son isomorphisme structural avec l'objet perçu².

Or, les images matérielles et mentales possèdent des caractéristiques générales communes et l'on peut supposer que les processus de constitution des images matérielles, dont on sait qu'ils sont profondément liés à notre activité perceptive, s'apparentent à ceux qui, sur la base de notre activité perceptive, produisent les images mentales ${ }^{3}$. Il y a plus de vingt ans déjà, Eco, nous l'avons dit (cf. ci-dessous), avait eu l'intuition que la relation d'analogie des icônes devait être fondée dans nos mécanismes perceptifs. Mais à l'époque, cette hypothèse n'a guère été entendue par les sémiologues dont la démarche, imprégnée de structuralisme, se trouvait entièrement centrée sur la découverte et l'identification des codes. Nous intégrerons donc au schéma 5 cidessus ces nouveaux éléments. Nous obtiendrons alors la représentation suivante :

1 IDEM, Image et cognition, op. cit., p. 64 et sv.

2 La notion d'objet perçu ne renvoit en aucun cas aux objets réels; il faut se souvenir de la définition de Reuchlin cité par le Groupe $\mu$ : "l'objet perçu est une construction, un ensemble d'informations sélectionnées et structurées en fonction de l'expérience antérieure, des besoins, des intentions de l'organisme impliqué dans une certaine situation " (GROUPE $\mu$, op. cit., p. 80).

3 Il s'agit toujours des processus de transformation, d'extraction et de réduction de l'information, de conservation de certaines relations structurales, topologiques, etc. Parmi ceux-ci, citons aussi la figuration qui consiste à extraire l'information figurable d'énoncés linguistiques en vue de construire des images mentales (M. DENIS, op.cit.): il fonde de la même façon l'activité de schématisation génératrice d'images matérielles. 




Schéma 5: Relations d'analogie (II)

Ce schéma demande quelques commentaires. Il faut savoir que l'objet perçu, peut être déjà une représentation, ce que tend à montrer le fait que dans de nombreuses expériences portant sur l'imagerie mentale les situations perceptives placent les sujets face à des représentations graphiques - des dessins- et non face aux objets réels. Le schéma pourrait donc contenir un sous-schéma enchâssé et le dispositif global indiquerait alors que l'on travaille en réalité sur des représentations de représentations. Ensuite nous avons introduit, suivant en cela Denis et de Vega, la notion nouvelle de modèle mental distincte de celle d'image mentale. Le modèle se distingue de l'image en ce qu'il est une représentation simplifiée accentuant de façon sélective les traits pertinents au regard d'une tâche à effectuer; il est donc construit sur les principes de l'activation sélective de la mise au premier plan. De plus, il peut incorporer d'autres informations de 
nature non visuo-spatiale, par exemple psychosociale ou relatives aux état émotionnels.

Or, c'est bien à cause de la similarité tant fonctionnelle que structurale entre les images mentales et les images matérielles que ces dernières peuvent dans certaines circonstances, par rapport à un certain public et pour certaines tâches, produire des effets, par exemple d'économie cognitive, ou d'autres plus complexes, tels que la supplantation. Ce dernier mécanisme a été mis en évidence et longuement analysé notamment par Salomon, Ausburn et Ausburn, Lynch, Smith, Donnay ${ }^{1}$. C'est le cas lorsque des systèmes symboliques, utilisés par exemple par le cinéma et la télévision, " sont tels que, au lieu d'exiger une transformation (comme lorsque deux points de vue sont juxtaposés et doivent être coordonnés), ils en réalisent au moins une partie pour le spectateur. Ainsi, le zoom modèle ou supplante ${ }^{2}$ l'opération que l'on doit effectuer intérieurement quand on passe d'un gros plan à un plan général. De même, le mouvement de la caméra autour d'un objet supplante le processus de coordination des points de vues"3. La supplantation intervient donc au moment où l'apprenant intériorise un mode de traitement de l'information externe, réalisé par certaines caractéristiques techniques, syntaxiques, etc., du langage audio-scripto-visuel plutôt que de devoir appliquer lui-même à une image mentale un processus identique.

1 Dans son article "Tendances de la recherche en télévision éducative" (Guide des Médias, Vidéos, film, photo et moyen audiovisuels, Suppl. 10, 1992), Donnay présente une synthèse de ces recherches. Le lecteur pourra se référer à la bibliographie de cet article.

2 En caractères italiques dans le texte.

3 G. SALOMON, "La fonction crée l'organe", Communications, 33, 1981, p. 86. 


\section{Schémas et images pédagogiques: le poids des sciences}

Les recherches cognitivistes ont donné naissance à de nombreuses études sur le rôle des représentations figuratives dans les processus d'apprentissage, tant en situation de laboratoire qu'en milieu scolaire. Étant donné la place qu'il occupe dans les textes scientifiques, dans la littérature de vulgarisation et en didactique des sciences (biologie, chimie), le schéma a fait l'objet d'une attention particulière de la part de nombreux chercheurs. Notons cependant qu'il existe une grande imprécision quant à la définition de l'objet étudié. Par exemple, les représentations figuratives qui sont au centre des recherches de Reid ${ }^{1}$, d'Astolfi' ${ }^{2}$ et d'Arnaud ${ }^{3}$ (1984) sont étrangement ressemblantes. Or, il s'agit pour le premier d'images, de diagrammes, de dessins au trait, pour le deuxième d'images et enfin pour le dernier de schémas. Nous conserverons cependant provisoirement ce dernier terme puisqu'il est le plus usité dans la littérature consacrée dont nous présentons ici une brève recension. Celle-ci fait apparaître l'énorme diversité des intérêts et des thèmes traités:

- l'utilisation du schéma comme instrument de formalisation des connaissances, c'est-à-dire comme langage spécifique d'exploration et d'expression scientifiques;

- l'apprentissage de leur lecture;

- l'adéquation du schéma à son contenu informationnel;

- la figurabilité des concepts scientifiques;

- la mise en correspondance entre les énoncés verbaux et les schémas ou le traitement image/texte;

- le rôle du schéma dans la mémorisation d'informations et dans l'acquisition des connaissances;

- la réalisation de manuels scolaires.

L'analyse de ces travaux permet cependant d'identifier deux orientations principales. Dans la première, le schéma est considéré comme une forme de représentation et de communication; il devient donc le centre d'une réflexion spécifique visant à comprendre ses modalités et son fonctionnement pour " mieux en saisir la nature et les

1 D. REID, Quelques investigations..., op. cit.

2 J.-P. Astolfi et al.., op. cit.

3 P. ARNAUD, "Forme et fonctions des éléments figuratifs dans la littérature didactique en Chimie", Bulletin de Psychologie, XLI, 386, 1988, p. 577-582. 
implications". On comprendra que dans cette perspective les rapports entre texte et paratextes passent au second plan. A ce courant se rattachent notamment des travaux récents portant sur les traitements cognitif et didactique des schémas et des graphiques et rassemblés dans un numéro de la revue Les sciences de l'éducation pour l'ère nouvelle ${ }^{2}$. On citera, à titre d'exemple des problématiques traitées, les travaux de Duval sur la pertinence des variables visuelles dans la représentation graphique des équations, ceux de Jelinski sur l'efficacité comparée des diagrammes circulaires et orthogonaux, ceux de Lerouge sur l'influence des représentations graphiques sur les significations et concepts mathématiques (effets de contamination et de contagion), ou encore ceux de Baillé et Maury sur les composantes sémio-pragmatiques déterminant le processus d'interprétation de graphiques élémentaires. On rappellera aussi les recherches plus anciennes de l'équipe des didacticiens des sciences expérimentales de l'INRP $^{3}$ ou d'Arnaud ${ }^{4}$ qui analysent les caractéristiques et les fonctions des schémas par rapport aux phénomènes, à la réalité observable qu'ils représentent. Remarquons cependant que les auteurs qui travaillent dans cette dernière perspective englobent souvent, sans faire réellement la différence: $1^{\circ}$ ) le processus de schématisation luimême qui correspond de la part du producteur à l'activité cognitive préalable à toute visualisation et au traitement graphique ${ }^{5} ; 2^{\circ}$ ) le schéma proprement dit, c'est-à-dire le produit de ce processus.

Le second ensemble de recherches prend comme objet les rapports de complémentarité entre l'expression verbale et non verbale d'une connaissance, entre le texte et le schéma, principalement dans une perspective d'extraction de l'information, d'interprétation et d'acquisition de connaissances scientifiques: les caractéristiques et les fonctions des schémas sont dans ce cas analysées en référence aux énoncés qui les accompagnent ${ }^{6}$. Les rapports entre le texte et le para-

1 P. DUCHASTEL et al, op. cit., p. 667.

2 J. BaIllé et S. MAURY, op. cit.

3 J.-P. ASTOLFI et al., op, cit.

4 P. ARNAUD, "Schémas et représentations...", op. cit.

5 C. Belisle et G. JouAnNAdE, La communication visuelle, Paris, Les Éditions d'organisation, 1988.

6 A. DREYFus \& Y. MAZOuZ, "L'utilisation judicieuse du langage des graphiques par des élèves de seconde dans le domaine de la biologie", in J. BAILlé \& S. MAURY, (Ed.) op. cit., p. 245-265; D. REID, "The picture...", op. cit.; IDEM, "Quelques investigations...", op. cit.; IDEM, "The Role of Pictures in Learning Biology", Journal of Biological Education, 24, 3-4, 1990, p. 161-172 et 251-258; J.-F. VEZIN et L. VEZIN, "Schématisation...", op. cit.; F. BASTIDE, "Iconographie des textes 
texte sont alors analysés par rapport à la réalisation d'une tâche d'apprentissage telle que la mémorisation et la restitution de l'information, la compréhension, etc. L'article de Peeck' constitue une synthèse bien documentée d'un certain nombre de ces recherches, notamment de celles de Barcharach, Carr et Mehner ${ }^{2}$, Jörg et Hörmann ${ }^{3}$ et Bernard ${ }^{4}$.

Mais qu'est-ce donc qu'un schéma ? Répondre à cette question au vu de la littérature n'est pas une tâche aisée car les auteurs, nous l'avons indiqué, ne prennent pas toujours en considération les mêmes objets, les mêmes contextes, les mêmes références. Aussi les définitions qui s'appuient sur l'analyse de réalités différentes sont-elles rarement comparables. Par exemple, il n'est pas toujours évident de savoir si l'instance référentielle du schéma est constituée par la réalité observable ou par l'énoncé verbal auquel se rapporte le schéma. Une autre confusion réside dans la définition de la fonction des schémas: celle-ci est tout autre selon que l'on considère le processus de schématisation lui-même ou les processus de lecture et d'interprétation. Dans le premier cas le schéma peut apparaître comme un instrument de formalisation et de connaissance du réel tandis que, dans le second, on le considérera comme le support d'une stratégie d'apprentissage et l'instrument d'acquisition de connaissances. Mais en ne maintenant pas la distinction entre ces deux points de vue, on assimilerait l'une à l'autre la sphère de la production du schéma et celle de sa lecture ou de son interprétation. Or, pour nous, il s'agit de deux problématiques qui doivent demeurer distinctes. Cette position méthodologique est par ailleurs corroborée par des études expérimentales: Maury, Janvier et Baillé 5 ont en effet pu montrer, dans une population d'élèves du premier cycle de l'enseignement secondaire français, que la construc-

scientifiques", Culture Technique, 14, 1985, p. 133-151; J.-F. VEZIN et L. VEZIN, "Illustration...", op. cit.;KIRBY, JURISICH \& MOORE, 1984; J. R. KIRBY, op. cit.

1 J. PEECK, "Increasing picture effects in learning from illustred text", in E. DE CORTE, op. cit., vol. $3, \mathrm{n}^{\circ} 3$, p. 227-238.

2 V.R. BARCharach, T.H. CARR et D.S. MEHNER, "Interactive and independent contributions of verbal descriptions to children's picture memory", Journal of Experimental Child Psychology, 22, 1976, p. 492-498.

3 S. JÖRG et H. HORMANN, "The influence of general and specific verbal labels on the recognition of labelled and unlabelled parts of pictures", Journal of Verbal Learning and Verbal Behaviour, 17, 1978, p. 28-37.

4 R.M. BERNARD, "Using extended captations to improve learning from instructional illustrations", British Journal of Educational Technology, 21, 1990, p. 215-255.

5 S. Maury, M. Janvier \& J. Baille, "Bar Diagram Processing Procedures", European Journal of Psychology of Mathematics Education, 5, 3, 1990, p. 293-308. 
tion et la lecture de graphiques donnaient lieu à des performances très différentes.

Une seconde difficulté provient du fait que les textes présentent une certaine imprécision terminologique. Selon les auteurs, les schémas se voient définis par leurs "caractéristiques", leurs "fonctions" ou leur "valeur" et les deux premiers de ces trois termes semblent parfois considérés comme des expressions équivalentes. Or, la nature des schémas nous semble pouvoir être définie par leurs caractéristiques tandis que leur fonction le serait par le rôle qu'ils jouent dans les processus auxquels ils participent. De ce point de vue, nous voudrions évoquer la classification des paratextes que propose Belisle ${ }^{1}$; l'auteur distingue sept types de paratexte dont le degré d'iconicité va décroissant, mais dont les fonctions sont exclusivement définies par rapport à cette échelle d'iconicité :

- la photographie, dont les principales fonctions sont: montrer, prouver, évoquer et proposer des éléments de motivation;

- les dessins, formes de représentation analogique en deux ou trois dimensions;

- les graphiques, dont les structures analogiques paraissent adaptées à la représentation de données quantifiées; nous rappellerons à ce propos le travail remarquable de Bertin $^{2}$ sur la morphologie de l'image graphique qui a longtemps servi de référence;

- les organigrammes, qui sont un type particulier de graphe utilisés pour représenter des relations structurées;

- les schémas qui, comportant seulement les traits essentiels de la figure représentée, sont utilisés pour la présentation et l'explication des objets ou des phénomènes complexes;

- les tableaux, qui sont constitués d'un ensemble d'éléments indifférenciés visuellement et dont l'information est extraite par une analyse des rapprochements et de comparaisons entre les éléments;

- les textes.

On le voit, en liant la fonction des paratextes à la définition de leur degré d'iconicité, il devient impossible de leur attribuer d'autres fonctions que celles liées à leur statut de signe et à leur qualité d'icone. Le degré d'iconicité des paratextes est plus que vraisemblablement corrélé avec certaines fonctions. Cependant, nous pensons

1 C. Belisle et G. JOUANNADE, op. cit.

2 J. BERTIN, Sémiologie graphique, Gauthier Villars-Mouton, Paris-La Haye, 1967; IDEM, "La graphique", Communications, 15, 1970, p. 160-185; IDEM, Le graphique et le traitement graphique de l'information, Paris, Flammarion, 1977. 
quant à nous que la seule façon de faire apparaître ces relations -entre nature des paratextes et fonctions- consiste à définir les uns et les autres selon des critères distincts, respectivement le degré d'iconicité et le fonctionnement discursif, car toute autre démarche produit un effet de miroir et une corrélation induite par le mode de définition de ces deux variables.

C'est pourquoi, même si les résultats paraissent contradictoires, la tentative de typologie des graphiques figurant dans des manuels scolaires d'histoire-géographie et de biologie-géologie ${ }^{1}$ nous paraît plus intéressante. Les auteurs procèdent à l'analyse des seuls "diagrammes à voir", selon la terminologie de Bertin ${ }^{2}$. On se souviendra que pour le cartographe, l'un des fondateurs de la sémiologie graphique, un diagramme est la représentation relationnelle de deux dimensions, de deux phénomènes, telle que "les correspondances dans le plan de représentation peuvent s'établir entre toutes les divisions d'une composante et toutes les divisions d'une autre composante "3. Un tableau à deux entrées, un graphique présentant deux axes orthogonaux $(x, y)$ illustrent parfaitement cette définition. Quelle est alors la différence entre les diagrammes "à lire " et ceux "à voir" ? Les premiers permettent simplement de faire correspondre de l'information alphanumérique à partir d'un tableau à double entrée, tandis que les seconds font apparaître des relations entre divers facteurs ou décrivent la répartition d'une ou plusieurs statistiques.

Sur la base de cette définition, les auteurs excluront de leur étude les cartes topographiques, les cartes politiques, les chroniques, les schémas anatomiques et les coupes géologiques. Ils analyseront les diagrammes à voir à partir de trois champs: l'observation des données (quantitatives, modales, ordonnées, etc.), le codage mathématique des données (calcul de moyennes et de fréquences, regroupement en classes, etc.) et, enfin, le traitement visuel selon la classification de Bertin en variables rétiniennes (taille, valeur, orientation, etc.). Une analyse factorielle des correspondances fait apparaître certains critères permettant de proposer une approche typologique en six familles bien

1 M. Janvier, R. Sabatier, J. Baillé \& S. Maury, S. "Essai de typologie des graphiques dans les manuels d'histoire-géographie et biologie-géologie des collèges", in J. BAILLÉ, \& S. MAURY, (Ed.), "Les représentations graphiques dans l'enseignement et la formation", Les sciences de l'éducation pour l'ère nouvelle, 13, 1993, p. 221-244.

2 J. BERTIN, Sémiologie graphique, op. cit.

3 Ibidem, p. 50. 
que celles-ci ne soit pas toujours nettement définies et que l'interprétation des résultats demeure mitigée:

Il ne semble donc pas possible de procéder à une taxinomie des graphiques bien que la plupart des graphiques soient voisins d'un ou de plusieurs types proposés. L'étude montre également le rôle perturbateur joué par le codage des variables observées qui peuvent conduire à des dessins semblables pour la représentation de situations très différentes ${ }^{1}$.

Quelles sont alors les caractéristiques attribuées à un schéma ?

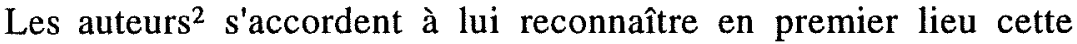
première caractéristique fondamentale: la capacité à isoler, au sein d'un phénomène pris dans sa globalité, certaines relations invariantes lui conférant alors un important pouvoir d'abstraction. C'est à cette caractéristique générale que nous rattacherons celle de "figuration simplifiée, fonctionnelle et modélisante du réel " proposée par Arnaud ${ }^{3}$. Le schéma implique en effet le dépassement de cas particuliers et débouche sur une activité de catégorisation ou de classification. Il doit donc être considéré comme une "expression figurative ayant valeur de généralité " au sens où Veròn dit des dessins schématisés des manuels d'anatomie, par exemple, qu'ils doivent être interprétés " comme le support d'un message associé à l'idée ou au modèle du corps humain dans un sens général " et qu'“ ils présentent ou proposent (par opposition à "affirment") une classe de choses et non un exemple particulier de quelque chose " 4 . Vezin ${ }^{5}$ situe quant à lui cette valeur de généralité entre le schéma et les énoncés du texte informatif correspondant et, du coup, fait de cette caractéristique une fonction: elle faciliterait l'utilisation des connaissances dans les activités de restitution, d'application et même de transfert.

La deuxième particularité des schémas identifiée par Vezinn, toujours dans le contexte des correspondances schémas/énoncés, découle directement de leur valeur d'abstraction; elle en est le corol-

1 Ibidem, p. 243 ,

2 Fr. Bresson, "Réflexions sur les systèmes de représentation", Sémiologie Graphique, 73/74, 1975, p. 7-13; J.-P. Astolfi et al., op. cit.

3 P. ARNAUD, "Schémas et représentations...", op. cit.

4 E. VERÒN, "L'analogique et le contigu", Communications, $n^{\circ} 15,1970$, p. 63.

5 J.-F. VEZIN \& L. VEZIN, "Schématisation...", op. cit.

6 Ibidem. 
laire: "la valeur de généralité favorise la sélection". Les schémas orientent l'activité d'étude vers les caractéristiques générales valables pour un ensemble d'objets ou de phénomènes et centrent l'attention sur l'essentiel. Dans ce cas, il s'agit donc bien d'une fonction et celleci porte sur l'activité de lecture et d'interprétation des énoncés que l'on souhaite voir réalisée par l'apprenant. Pourtant, soulignons que la généralisation et la sélection sont aussi deux activités cognitives qui fondent le processus de schématisation: elles vont de pair puisque généraliser veut dire abstraire à partir des phénomènes observés des régularités, donc sélectionner les caractéristiques valables pour un ensemble constituant une classe. Mais dans cette seconde acception, généralisation et sélection appartiennent nettement à la sphère de production des schémas. Pourtant rien ne permet d'affirmer que ces deux termes -généralisation et sélection- recouvrent des opérations identiques dans la sphère de production des schémas et dans celle de leur interprétation. Autrement dit, le processus de schématisation qui prend la réalité observable pour objet n'est pas nécessairement assimilable à celui d'extraction d'informations qu'induiraient, à partir des énoncés, les schếmas.

La troisième caractéristique du schéma est d'assurer une médiation entre un concept abstrait et la constitution d'une image mentale de ce concept ${ }^{2}$. Sa nature figurative et analogique rendrait donc possible la concrétisation de concepts ou de phénomènes difficiles à représenter, ce qui constitue habituellement le rôle de l'imagerie scientifique ${ }^{3}$. Le schéma apparaît alors comme un instrument de formalisation et de représentation du savoir et sa nature analogique le rendrait proche de la métaphore du langage verbal ${ }^{4}$. A cette fonction de concrétisation, nous rattacherons une valeur d'objectivations: "Le schéma conduit à une vue d'ensemble grâce à son symbolisme figuratif qui lui donne aussi plus ou moins l'apparence d'un objet "6; contrairement à l'image mentale, le schéma est une représentation, une figuration matérialisée. Cette valeur d'objectivation par ailleurs varie: elle est d'autant plus grande que le schéma apparaît davantage comme un objet réel, qu'il

1 J.-F. VeZin \& L. VeZIN, "Illustration...", op. cit.; C. Belisle \& G. JouANnADE, op. cit.

2 P. ARNAUD, "Schémas et représentations...", op. cit.

3 D. JACOBI, "Figures et figurabilité...", op. cit.

4 P. ARnAUd, "Schémas et représentations...", op. cit.

5 GiLET, 1980; J.-F. VEZIN \& L. VeZIN, "Schématisation...", op. cit.

6 J.-F. VeZIN \& L. VEZIN, "Illustration...", op. cit., p. 659. 
est descriptif ou qu'il présente une vue d'ensemble, autrement dit qu'il est réaliste et ressemblant. Ensuite, elle rend le schéma plus proche d'une réalité connue et facilite l'interprétation de données en fournissant des points de repère. Vezinn a montré par ailleurs qu'une plus grande valeur d'objectivation facilite la rétention de l'information et, de façon générale, une meilleure mise en relation des schémas avec les énoncés verbaux. Enfin l'hypothèse de Gilet selon laquelle l'objectivation rend possible la représentation imagée pourrait être interprétée aujourd'hui dans le sens où elle favoriserait la création d'un modèle mental transitoire, cristallisant le pouvoir imageant des énoncés auxquels le schéma se réfère. Et c'est bien pour cette raison que le schéma pourrait servir de support figuratif au traitement de l'information.

Ensuite, beaucoup de schémas scientifiques présentent une valeur synoptique qui constitue leur quatrième caractéristique: différents éléments essentiels peuvent être présentés simultanément, ce qui n'est possible ni dans le réel ${ }^{2}$ ni dans la succession linéaire qu'imposent le langage verbal ou, au niveau conceptuel, le codage propositionnel ${ }^{3}$. Cette valeur synoptique procède d'un double mouvement d'abstraction et de concrétisation: un schéma accentue la symbolisation des relations entre les éléments, privilégiant les structures par rapport aux formes sensibles et matérielles, et permet de regrouper dans la même représentation différents éléments abstraits habituellement isolés ainsi que leurs relations ${ }^{4}$. Les représentations des manuels scolaires constituent de bons exemples de ce point d'équilibre, de ce compromis entre ces deux tendances complémentaires. Mais ces avantages si souvent mis en évidence appellent des restrictions, fort bien formulées par Arnaud 5 . Le processus de schématisation, par sa nature même, est susceptible d'entraîner un biais important: l'appauvrissement et du réel par sursimplification et des concepts abstraits par une représentation trop simpliste.

Enfin, Vezin ${ }^{6}$ attribue aux schémas une valeur d'économie cognitive. Grâce à leur valeur synoptique, ils permettent de considérer

1 J.-F. VeZIN, Complémentarité du verbal et du non-verbal dans l'acquisition des connaissances, Paris, CNRS, 1980.

2 J.-P. ASTOLFI et al., op. cit.

3 J.-F. VEZIN \& L. VEZIN, "Schématisation...", op. cit.; J.-F. VEZIN, "Mise en relation...", op. cit.

4 J.-P. Astolfi et al., op. cit.

5 P. ARNAUd, "Schémas...",op. cit.

6 J.-F. VeZIN \& L. VeZIN, "Schématisation...", op. cit. 
en une même appréhension des caractéristiques, des relations que le symbolisme verbal ne peut exprimer qu'à travers plusieurs énoncés. Ils expriment donc de façon synthétique un ensemble de données, allégeant ainsi la charge mnémonique pour l'apprenant. Cet allégement sera d'autant plus important que le schéma montre, en un ensemble, les relations entre les données dont l'unité apparaît comme une forme pouvant être retenue en tant que telle: il favoriserait donc la création ou l'activation de modèles mentaux. Nous rattacherons à cette dernière caractéristique le picture superiority effect (PSE), effet de supériorité lié à l'image, étudié notamment par Reid dans les manuels de biologie'. Pourtant, on ferait fausse route en croyant que celui-ci se réalise dans toutes les circonstances. Reid conclut à l'existence d'un PSE, mais uniquement dans certaines conditions: par exemple lorsque ce qui doit être appris se trouve de façon redondante dans l'image et dans le texte, lorsque les informations contenues dans le texte et l'image sont de même nature, lorsque la tâche d'apprentissage est basée sur la mémorisation plutôt que sur des activités cognitives plus complexes, telles la compréhension, ou encore quand les schémas sont présentés à des élèves de capacité moyenne ou au-dessus de la moyenne. Que la redondance entre l'information visuelle et textuelle soit une condition essentielle pour l'observation du PSE a été plusieurs fois confirmé par les recherches expérimentales:

(...) many of the studies reviewed show that retention of text content is improved by illustrations, but mainly, and perhaps exclusively, inasmuch as the illustrations concerned actually depict what it says in the text ${ }^{2}$.

Dans les autres cas, les résultats paraissent contradictoires: on constate un effet de facilitation ${ }^{3}$ ou, au contraire, aucun effet n'est observé4.

L'on sait aussi que la qualité de l'exploitation d'un diagramme ou d'une illustration est déterminée par la connaissance préalable qu'a

1 D. REID, “The picture...", op. cit; IDEM, "Quelques investigations...", op. cit.

2 J. PEECK, "Increasing picture...", op. cit., p. 227.

3 Notamment RuSTED et HodgSON, 1985 cité par J. PEECK, ibidem.

4 Notamment J. PeECK \& M.W. JANS, "Delayed retention of orally presented text with pictorial support", British Journal of Educational psychology, 57, 1987, p. 412-416 ou J.R. KIRBY, op. cit. 


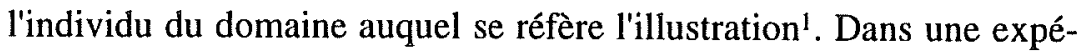
rience récente mettant des météorologues et des non météorologues face à des tâches nécessitant des savoir-faire caractéristiques de cette discipline, Lowe constate la supériorité des premiers sur les seconds et de conclure:

If a diagram intended as an aid to learning leads to the construction of a mental representation that fails to capture properly the aspects of the subject matter wich have semantic signifiance, it is unlikely that the desired learning will be facilitated. A mental representation of the contents of the diagram in largely visuo-spatial terms would make it very difficult for a learner to gain the benefits that Mayer and Gallini (1990) suggest can flow from the capacity of diagram to support more effective processing based upon the running of mental models ${ }^{2}$.

Nous terminerons enfin l'examen de ces recherches par deux remarques. Premièrement, tous les travaux le montrent, la lecture, la compréhension et l'exploitation des schémas, comme celles de toute image ou même des textes, ne sont ni évidentes ni spontanées": "Les images ne sont pas pour les élèves plus transparentes que les textes et il importe d'en répertorier les obstacles, les pièges et les doubles sens. Aussi est-il nécessaire de mettre en oeuvre des stratégies d'apprentissage appropriées afin de favoriser une meilleure appropriation de ces systèmes de représentation par les apprenants. Parmi ces stratégies, citons Janvier :

L'enseignement de l'interprétation de graphiques devrait naturellement aller du simple vers le complexe en jouant sur diverses variables didactiques, celles concernant la nature des situations étudiées, celles qui relèvent des traitements mathématiques des variables observées et celles qui relèvent du traitement visuel proprement dit, en distinguant

\footnotetext{
1 A. JELINSKI, "Diagramme circulaire ou orthogonal: une efficacité différente des images graphiques dans la transmission des informations", in J. BAILLÉ \& S. MAURY, (Ed.) op. cit., p. 36-56; J. BAILlE, op. cit. et R.K. LOWE, op. cit.

2 R.K. LOWE, op. cit., p. 177.

${ }^{3}$ L. J. Issing, "Learning from Pictorial Analogies", European Journal of Psychology of Education, 4, 1990, p. 489-499 dans D. JACOBI, "Économie visuelle et tension narrative des représentations graphiques", in J. BAILLÉ, \& S. MAURY, (Ed.), op. cit., 1-3, p. 267-284; D. REID, "The Role of Pictures...", op. cit.; J.-P. Astolfi et al., op. cit.
} 
soigneusement les traitements adaptés au relationnel de ceux qui sont adaptés au mesurable ${ }^{1}$.

Plus catégorique est la proposition de Lowe:

This would suggest that teachers may need to discriminate between different types of diagrams and see abstract diagrams as a potential learning problem in themselves rather than as a solution to such problem ${ }^{2}$.

Ce qui est vrai des schémas en général l'est aussi des graphiques et des tableaux. Chacun de ces deux modes de représentation demanderaient en effet des capacités spécifiques et il semblerait que, parmi les élèves, les capacités à exploiter l'un et l'autre ne soient pas corrélées: il y aurait nettement deux styles cognitifs et rares seraient les élèves bicognitifs, c'est-à-dire capables de traiter l'information efficacement dans l'un et l'autre de ces deux modes de représentation ${ }^{3}$. Dans cette perspective, rappelons aussi qu'il existe de plus en plus de logiciels d'apprentissage à la lecture et à l'interprétation des graphiques et des tableaux. Enfin, toujours d'après les résultats observés par ces deux derniers auteurs, la capacité d'utilisation des tableaux et des graphiques par les élèves serait sous-utilisée au regard de leur capacité potentielle. Nous rapprocherons cette observation de celles de Weidenmann ${ }^{4}$ et de Peeck ${ }^{5}$ pour lesquels, même si l'on a pu démontrer expérimentalement dans certaines conditions les effets positifs des illustrations sur l'apprentissage, la pratique scolaire est loin d'en avoir intégré les avantages:

Learners will often deal with illustrations in a superficial or otherwise inadaquate way, with the result that quite often, as Weidenmann puts it, "good picture fail" in achieving any contribution to the instructional process ${ }^{6}$.

Notre seconde remarque porte sur la valeur de généralité de schémas. D'aucuns ont cru pouvoir en faire un critère de distinction entre schéma et illustration. Vezin et Vezin, définissant les caractères

1 M. JANVIER et al., op. cit., p. 244.

2 R.K. LowE, op. cit., p. 177.

3 A. DREYFus \& Y. MAZOUZ, op. cit.

4 WEIDENMANN, 1989, cité par J. PEECK, "Increasing picture...", op. cit.

5 Ibidem.

6 Ibidem, p. 228. 
formels de l'illustration et du schéma, défendent l'idée que la première représente un objet particulier, "un réel de référence tel qu'il apparaît " alors que dans le schéma l'objet dessiné serait représentatif d'un ensemble ${ }^{1}$. Autrement dit, l'illustration demeurerait enracinée dans la réalité référentielle concrète tandis que le schéma ouvrirait les portes de l'abstraction et de la classification. Or, il n'est pas simple d'opérer une telle distinction. En effet, le degré de ressemblance-d'iconicitédes illustrations et des schémas est variable: ces auteurs distinguent par ailleurs eux-mêmes des schémas descriptifs, plus proches du concret, et des schémas abstraits mettant en évidence les principes sous-jacents à la réalité représentée. De plus, comme le schéma, l'illustration se voit souvent utilisée pour visualiser un objet prototypique d'une classe: dans le cadre de l'apprentissage des langues, le dessin d'une étoile ou d'un canard ne désignent pas une étoile ou un canard particulier; tout au contraire ils sont là comme support, comme signifiant visuel, d'un signifié, d'une classe conceptuelle: "l'étoile", "le canard".

On observerait donc des usages, des fonctions et des rôles différents attachés cependant au même type de représentation (l'illustration par exemple). Inversement, la même fonction pourrait être assumée par plusieurs représentations types différentes (l'illustration et le schéma par exemple). Certains auteurs ont porté leur attention sur ce dernier aspect tout en soulignant la difficulté à associer à un schéma une fonction déterminée, chaque fonction pouvant donner lieu à une lecture différente du même schéma ${ }^{2}$. Dans le même ordre d'idées et à propos de l'analyse des fonctions des schémas, Arnaud affirmait que chacune d'elles - moyen d'expression scientifique, figuration et modélisation du réel, médiateur entre le concept et la représentation mentale de celui-ci-donnait lieu à trois effets spécifiques: effets de description, de compréhension et d'assimilation, effets cependant difficilement isolables.

1 J.-F. VEZIN \& L. VEZIN, "Illustration...", op. cit., p. 655-656.

2 J.-F. VEZIN \& L. VEZIN, "Schématisation...", op. cit. 


\section{Conclusions}

L'analyse de ces différents paradigmes de recherche met en évidence la difficulté d'établir une typologie des fonctions cognitives et pédagogiques des images didactiques et souligne combien la définition et la représentation des paratextes demeurent très souvent ambiguës. Si l'on cherche à articuler réellement la théorie psychologique des représentations mentales et une théorie sémiopragmatique des représentations matérielles, il faut absolument rendre les terminologies et les concepts compatibles, éviter les confusions qui, nous l'avons vu, sont nombreuses. En toute rigueur, une description scientifique devrait dès lors distinguer les différents niveaux d'analyse:

1. les capteurs sensoriels et la perception;

2. les supports d'affichage et de présentation;

3. les modes de représentation et la nature sémiotique des différentes représentations;

4. les formes de codes et de traitement de l'information au plan cognitif.

A chacun de ces niveaux devrait correspondre une désignation propre, respectivement:

1. multimodal renvoyant aux différentes modalités perceptives et donc au canal;

2. audio-scripto-visuel selon le mode de présentation;

3. analogique et symbolique en fonction de la nature du signe et des formes de représentation;

4. propositionnel, analogique et phonologique par rapport au mode de codage mnémonique.

Ensuite, une théorie des paratextes reconnaîtra certes la diversité des formes de représentations, analogique et symbolique, mais la considérera comme l'un de ses critères d'analyse. Il n'est pas sûr en effet que la recherche d'échelles d'iconicité, problématique qui s'enracine pour l'essentiel dans une théorie du code, constitue l'approche la plus fructueuse. Dans notre étude déjà citée sur les paratextes dans les manuels d'économie et de biologie', nous avons partiellement échoué sur ce point: il nous a été impossible d'établir une classification fine des paratextes selon leur degré d'iconicité puisque certaines catégories se sont avérées inutiles ou statistiquement non pertinentes. Le nombre de degrés dans une échelle d'iconicité a

1 D. PERAYA \& M.-C. NySSEN, “Les paratextes...", op. cit. 
donc pu être considérablement réduit, sans que l'analyse ne s'en trouve affaiblie, tout au contraire. De plus, le type de représentation, globalement anologique ou symbolique, ne permet pas de caractériser à lui seul une forme prototypique de discours. Les manuels de biologie et d'économie se distinguent par l'association de trois variables descriptives: le type de représentation, mais aussi la fonction du paratexte et la façon dont il lui est fait référence dans le texte même. Enfin, l'association des variables "représentation" et "fonction" semble stable indépendamment des disciplines considérées: on observerait toujours les configurations "analogique-fonction de représentation" et "symbolique-fonction d'information". Enfin, il semble difficile de ne pas tenir compte des facteurs exogènes, par exemple la tâche que doit accomplir l'apprenant.

A ce propos, il est étonnant que dans les nombreux manuels consultés il ne soit quasiment jamais fait allusion au traitement de la plage visuelle par l'apprenant. Des points de vue tant de la pratique pédagogique que de la théorie de l'apprentissage, il semblerait pourtant utile de donner au lecteur des consignes précises afin d'orienter la lecture des plages visuelles et d'en favoriser l'appropriation à l'occasion d'une tâche. L'utilisation et l'exploitation des paratextes irait de soi, comme si les élèves possédaient naturellement la capacité -le don ?- de traiter l'information présentée sous une forme audio-scripto-visuelle. Or, toutes les recherches que nous avons citées tendent à prouver le contraire. On redécouvre donc aujourd'hui, sous l'impulsion du traitement informatique de l'image, ce que l'audiovisuel classique avait fait apparaître voilà près de 30 ans: une alphabétisation, une éducation aux représentations visuelles, sont indispensables si l'on veut que les plages visuelles deviennent un véritable outil d'apprentissage, une technologie intellectuelle. 